\title{
EL PAPEL DE LOS ESQUEMAS EN LA LECTURA
}

\author{
THE ROLE OF SCHEMATA IN READING
}

\section{Beatriz Giudici Fernández}

\section{ABSTRACT}

Reading is a complex activity ruled by the human brain in which previous mental schemata play a key role. When reading, a series of schemata or «screens» are activated simultaneously while the reader accepts or rejects the options that are presented to him. Several schemata remain open at the same time, while others are closed, restructured or rejected. It is possible that schema theory may enable us to understand a bit more a process in which memory, recognition, experience, previous readings, etc. have an outstanding role in order to decipher a text, that is to say, to read.

Keywords: Reading, schemata, neurology, sociolinguistics, applied psychology

\section{RESUMEN}

La lectura es una actividad compleja dominada por el cerebro humano en la que juegan un papel primordial los esquemas mentales previos. Al leer se activan simultáneamente una serie de esquemas o «pantallas», que se activan/desactivan sucesivamente a medida que el lector acepta o rechaza las opciones que se le presentan. Varios esquemas se mantienen abiertos al mismo tiempo, a la par que otros se cierran, recomponen o descartan. Es posible que la teoría 
de los esquemas arroje luz sobre un proceso en el que la vista, la memoria, el reconocimiento, la experiencia, las lecturas previas, etc., tienen un papel primordial a la hora de descifrar un texto, es decir, de leer.

Palabras clave: Lectura, esquemas, neurología, sociolingüística, psicología aplicada.

Fecha de recepción 24 de junio de 2019.

Fecha de aceptación: 5 de agosto de 2019.

Cómo citar: Giudici Fernández, Beatriz (2019), «El papel de los esquemas en la lectura», en Actio Nova: Revista de Teoría de la Literatura y Literatura Comparada, 3 (2019): 166-193.

DOI: https://doi.org/10.15366/actionova2019.3.008 


\section{1. ¿QUUÉ ES LA LECTURA?}

Desde la antigüedad clásica hasta el Renacimiento, dos son las teorías que intentaron explicar el mecanismo de la lectura: la intromisión, según la cual una cualidad presente en el ojo humano permite aprehender el objeto, y la extromisión, para la cual es el objeto externo en sí quien envía la información pertinente. La teoría de Leonardo da Vinci constituyó un enorme paso hacia delante, ya que de alguna manera aunó ambas posturas al considerar que la mente era una especie de pequeño laboratorio donde lo aprehendido por los sentidos, es decir las percepciones, se convertían en impresiones en esa 'rete mirabile' -el cerebro-, para luego ser canalizadas por el centro del sentido común y transformadas por una o varias facultades (por ejemplo, la memoria) bajo la influencia del corazón (Manguel 2005: 77). Con todo, el dilema persistía. ¿Es el lector quien captura las letras, como plantearon en su día Euclides y Galeno (intromisión) o, por el contrario, son éstas quienes vienen en busca de nuestros sentidos, como defendieron Epicuro y Aristóteles (extromisión)? Para Leonardo y sus contemporáneos, la clave se hallaba en un libro escrito en Egipto en el siglo XVI por el erudito de Basora Hasan Ibn-al-Haytan, conocido también como Alhacén. En él, Haytan, siguiendo a Aristóteles, defendía que las cualidades de lo que vemos entraban en el ojo por el aire. Sin embargo, distinguió entre la mera sensación, que no es más que un acto inconsciente e involuntario, de la percepción, que constituye un acto voluntario de (re)conocimiento que permite al sujeto trascender lo que ve y descifrarlo, es decir, leer. Pero cómo se produce esto seguía siendo un misterio.

Habría que esperar dos siglos hasta que Roger Bacon, en el siglo XVIII, considerara que el proceso de ver es algo activo y bidireccional, ya que para él la imagen entra en el ojo y es captada por lo que denominaba 'las facultades visuales' del mismo (Manguel 2005: 80).

A pesar del avance teórico que implicaban las teorías de Haytan y Bacon, el mecanismo por el cual la lectura se convertía en percepción seguía careciendo de respuesta, lo mismo que el papel que jugaban en este proceso la vista, la memoria, el reconocimiento, la experiencia, etc. Estos autores sabían, además, que la lectura implicaba coordinar una serie de habilidades y estaba afectada por el tiempo.

Un siglo más tarde, una nueva ciencia, la neurolingüística, descubre que el hemisferio izquierdo del cerebro deviene el hemisferio dominante para (des)codificar el lenguaje, hecho que se inicia cuando el ser humano es expuesto al mismo. Es decir, el hombre es biológicamente capaz de almacenar, descifrar y recordar (Platón) sensaciones y el propio 
lenguaje. Esta capacidad no se refiere sólo a la lectura, sino que el habla evoluciona de igual modo: 'descubrimos' una palabra y 'emitimos' una palabra porque el objeto o idea que representa ya está en nosotros de forma innata. Pero este proceso de la lectura como (re)conocimiento no es automático, ya que no basta con tener dicha capacidad; es necesario aprender a leer.

En el siglo XX, el profesor Lecours concluye, al igual que Haytan, que leer implica, por una parte, 'ver' la palabra y, por otra, 'considerarla' de acuerdo con la información conocida. Para Lecours, «es como si la información que los ojos reciben de la página viajara por el cerebro a través de una serie de conglomerados de neuronas especializadas, localizadas en secciones determinadas del cerebro, efectuando funciones específicas. Aún no sabemos en qué consiste exactamente cada una de esas funciones» (Manguel 2005: 86).

A propósito del habla, el neurólogo Oliver Sacks cree que ésta tiene que ver con algo más que con las palabras; de hecho, cree que está ligada a la elocución, es decir, a las palabras necesarias para el discurso. Lo mismo podría decirse de la lectura. Según Manguel, «siguiendo el texto, el lector extrae su significado mediante un método muy complicado de significados aprendidos, convenciones sociales, lecturas anteriores, experiencias personales, y gustos individuales». Es decir, que para extraer un mensaje «debo aprehender el sistema [ ... ] y luego reconstruir el código de signos mediante una cadena de neuronas que elabora la información en mi cerebro y que da al texto algo que depende de quién soy y cómo he llegado a serlo» (Manguel 2005: 89).

\section{TEXTO Y DISCURSO}

Es innegable la aportación de Hjelmslev a la doble articulación de la lengua propuesta por Saussure, ya que, al dotar de forma y sustancia a los conceptos de significante y significado (o expresión y contenido respectivamente según la terminología de Hjelmslev) propuestos por Saussure, esto permite un análisis más profundo del concepto de signo y abre las puertas al estudio de un fenómeno tan útil para el análisis literario como es la connotación. Según el modelo de Hjelmslev, la física sería la encargada del estudio de la sustancia de la expresión y del contenido, la fonética, de la relación entre la forma y sustancia de la expresión, la lingüística, de la forma de la expresión y del contenido, y la semántica, de la relación entre la forma y sustancia del contenido. 
Así, el signo, entendido como la forma de la expresión y del contenido, es signo de algo, es decir, signo de la sustancia de la expresión y del contenido. Esta segunda aproximación al signo es más fácil cuando se aplica a unidades menores, tales como las palabras o frases, pero resulta más complicada en contextos de mayor envergadura, como las obras literarias, donde el 'sentido' proviene de una complicada red de asociaciones y connotaciones, que se superponen al valor denotativo del conjunto.

Un texto, entendiendo por tal lo que el sujeto dice a su interlocutor con un propósito comunicativo, no se da jamás en el vacío, sino que es necesario tener en cuenta tanto la situación en la que se emite como el co-texto para proceder a su interpretación (Malinowski, Firth, Halliday). Es más, para que dicha interpretación sea fructífera y no de lugar a error han de tenerse en cuenta todos los elementos que confluyen para garantizarla. Es decir, que para proceder al análisis del significado (o significados) del texto hay que atender al discurso, es decir a aquellos elementos extratextuales, por lo general de índole cultural, que permiten su desambiguación.

Halliday propone un modelo de análisis textual a partir de tres categorías para estudiar los aspectos situacionales de la lengua en uso, a saber, campo, modo y 'estilo' ('field, 'mode' y 'tenor'). La primera permite saber de qué se está hablando y la segunda, si se trata de un texto para ser hablado, leído, declamado, etc., mientras que la última analiza la relación entre los participantes, entendiendo por esto no sólo el grado de formalidad y informalidad entre los interlocutores ('politeness'), sino -y esto es de suma importancia para el análisis literario- lo que los mismos asumen que es sabido por los demás. Esta asunción mutua también se da en la vida cotidiana; por ejemplo, cuando un abogado habla con sus colegas lo hace de distinta forma de lo que lo haría si estuviera hablando con un lego. En literatura dicha asunción de conocimientos trasciende el significado unívoco y los otros significados posibles, así como las connotaciones que el texto pueda tener en la 'vida real' y abarca ese otro significado superpuesto pero verosímil que aporta una historia inventada.

Sin embargo, la teoría de Halliday ha sufrido críticas, en concreto por parte de aquellos que le achacan que su modelo se centra en el análisis textual y no tiene en cuenta el discurso, es decir, la información extra textual, para decodificar el mensaje.

Entonces, ¿qué permite a los participantes interpretar no sólo lo que se dice sino lo que está implícito y no se menciona abiertamente? Para Grice (1975), el discurso no es una mera colección de comentarios inconexos, sino una serie de 'esfuerzos cooperativos' en los que los participantes reconocen un propósito común o una serie de propósitos o, al menos, 
una dirección mutuamente aceptada. A este esfuerzo mutuo lo llama implicación conversacional'. Para que éste tenga lugar, han de darse una serie de condiciones, a saber: cantidad, calidad, relación y manera o forma. Según la primera, el hablante da la cantidad de información necesaria -ni más ni menos-, mientras que la segunda implica que su aportación debe ser verdadera. La tercera característica, la relación, tiene que ver con la relevancia del mensaje y la última, la manera o forma, remite no a lo que se dice, sino a cómo se dice, es decir, a la claridad. Además, esta última característica es imprescindible no sólo para la conversación, sino para todo tipo de conducta interactiva.

Con todo, no siempre suelen darse discursos 'puros’ según el modelo de Grice, sino que, por el contrario, en innumerables ocasiones los interlocutores pueden: 1) no respetar una máxima, con lo cual es posible que el mensaje sea equívoco, 2) decidir no seguir el principio cooperativo, 3) enfrentarse a un choque de las máximas arriba mencionadas (por ejemplo, el ajustarse a una de ellas puede implicar no seguir otra) y 4) no cumplir abiertamente con una máxima y usarla para generar una implicación.

La teoría de Grice ha tenido detractores, como, por ejemplo, Sperber y Wilson (1986), para quienes el principio de relevancia engloba las cuatro categorías arriba mencionadas.

Volviendo a la pregunta formulada con anterioridad, la incógnita sigue siendo qué permite a los interlocutores acceder a 'eso otro' que no se menciona explícitamente pero que ambos entienden. ¿Qué permite, entonces, al lector no sólo comprender que esta imitación creíble de la realidad llamada literatura no es un calco de la misma, sino una recreación, una invención verosímil, sino extraer uno o varios sentidos de la historia que lee? Debe de haber algo que le permita acceder a -como decía tan poéticamente Ibn-al-Haytan- «aquello que la escritura sugiere mediante indicios y sombras» (Manguel: 2005: 91).

\section{SCHEMATA}

Muchos han sido los intentos de explicar de forma sistemática el papel que desempeña la información previa en el uso del lenguaje, en especial en la comprensión del mismo. Gran parte de estos trabajos provienen de la psicología aplicada y de la inteligencia artificial, un campo que relaciona la psicología y la lingüística con la informática. Pero el tema ha interesado también a sociológicos, etnógrafos y sociolingüistas. 
En la década de 1960, el psicólogo norteamericano David Rumelhart (1984: 2) se planteó averiguar cómo las neuronas procesan la información, fenómeno que los biólogos abordaban a partir del estudio del papel que juegan en ello las neuronas y el tejido cerebral, mientras que los psicólogos se centraban en procesos más abstractos como el razonamiento y las estrategias de aprendizaje. Años más tarde, Rumelhart y Mc. Clelland argumentaron que el lenguaje, como la mayoría del conocimiento, se basaba en la memoria y estaba representado en el cerebro mediante grupos de asociaciones entre elementos de sonido y significado. Dentro de su enfoque de redes neuronales en relación con el lenguaje y la cognición, Rumelhart acuñó el concepto de 'teoría de los esquemas' (schema theory), que, según él, es «básicamente una teoría acerca del conocimiento; una teoría acerca de cómo se representa el conocimiento y de cómo esta representación facilita ese conocimiento de una manera concreta».

Parece ser que el término fue usado por primera vez por Bartlett en 1932, pero Carrell (1983) opina que ya se halla en Kant. Otros autores prefieren el término 'frame' (marco) (Minsky: 1975), 'plan' (plan) (Schank \& Abelson: 1977) o 'scenario' (escenario) (Sanford \& Garrod: 1981) para referirse al mismo fenómeno, es decir, al mecanismo mediante el cual el cerebro almacena información y la activa cuando es necesario. Pongamos, por ejemplo, la palabra 'flor'. Minsky sugiere que en nuestra memoria tenemos archivada la representación de una flor, lo cual constituye una especie de archivo que él llama 'marco'. Esta representación tiene una serie de 'casilleros' que albergan términos/conceptos tales como pétalos, corola, estambres, fragancia, etc. Según Minsky, cuando vemos una flor se activa nuestro marco mental de/para 'flor', que puede ajustarse según la información recibida. De igual modo, el conocimiento lingüístico puede archivarse según categorías: léxico, morfología, género, etc. Lo mismo ocurre con otros campos. Por su parte, Fillmore utiliza el concepto de 'marco' para referirse a las representaciones semánticas dentro del conocimiento lingüístico, mientras que Sanford y Garrod prefieren el término ‘escenario' y creen que para comprender un texto escrito el sujeto tiene que activar el 'escenario' mental adecuado.

Carrell ha contribuido a la teoría de los esquemas con una distinción de suma importancia, ya que distingue entre los esquemas formales y los de contenido (formal \& content schemata). Los primeros incluirían la forma de las historias, las estructuras retóricas, etc., mientras que los segundos tendrían que ver con situaciones sociales, ritos, etc. Los esquemas de contenido son más parecidos al concepto de esquema en Rumelhart, Labov y Waletsky, 
para quienes una historia no es sólo una historia novelada, una historia literaria, sino que también debe entenderse por tal a todo tipo de narraciones, incluidas las que se mencionan en una conversación en la vida cotidiana. Tomemos el conocido ejemplo de Sacks: el bebé lloró; su madre lo cogió, lo que, sin duda, constituye una historia y está enmarcado en un tipo de esquema: problema-solución. Es decir, que un esquema sería una representación del conocimiento del mundo (Brown y Yule: 1983).

Es posible que el ejemplo de Sacks arriba mencionado sea comprensible en todo el mundo. Puede, también, hacerse extensivo a situaciones tales como una transacción comercial, donde se entiende que alguien compra algo que alguien vende, o una boda, en la que se espera que ha de haber un novio, una novia, parientes e invitados, e incluso un rito. Se podría argumentar que estas situaciones sociales son comunes a distintas culturas y que incluso resisten el paso del tiempo, y que, por lo tanto, han de ser entendidas en cualquier parte del globo. Pero la primera, la transacción comercial, podría no ser entendida como tal en una cultura que no tenga relaciones de compra-venta y malinterpretarse como un trueque, por lo que la mención del dinero podría resultar desconcertante. Y la segunda, la boda, podría crear expectativas que no se cumplen. Por ejemplo, si leemos que llega la novia vestida de blanco y se oye música nupcial, lo más seguro es que pensemos en Mendelsohn. Sin embargo, podemos no entender, a menos que pertenezcamos a esa religión, por qué en una boda judía se rompe un vaso o por qué los novios se sitúan bajo palio (la jupâ). Tendríamos, pues, un marco común en el que aparecen los elementos esperados: los novios y sus familiares, el intercambio de anillos, la persona que oficia el rito o sacramento, y elementos que distorsionan las expectativas y que, por ende, pueden hacer que no comprendamos o que distorsionemos el mensaje. Por ejemplo, en la boda judía el sacramento bajo palio recuerda a la casa común que la pareja habitaba antiguamente y la ruptura del vaso conmemora, por una parte, la destrucción del templo de Jerusalén hace más de dos mil años y, por otra, la ruptura de sus respectivas almas, aunque ambas rupturas están relacionadas. El templo de Jerusalén representaba la unión del cielo y la tierra, de lo ideal y lo real, de Dios y la creación. En lo que respecta al alma de los novios, ambos eran una sola alma antes de nacer, pero al ver la luz, su alma se rompió en dos: femenino y masculino. La boda implica que sus respectivas almas se han reencontrado y unido para siempre, pues el único antídoto contra la fragmentación es la unidad. La boda ha unido lo que antes estaba fragmentado en dos: tanto las respectivas almas de los contrayentes como el templo, enmendando así dos antiguas rupturas que, en el fondo, son una sola. 
Esto nos lleva a la conclusión de que, aunque es posible que haya esquemas comprensibles por todo el mundo, como el de Sacks antes analizado, en general los esquemas están determinados culturalmente. Y es posible que también lo estén temporalmente, pues el tiempo cambia los usos y costumbres. Es interesante recordar aquí que Steiner señala con acierto que el acceso al significado parcial o total de una obra perteneciente al pasado de nuestra lengua implica un proceso de traducción, pues el contexto socio-cultural que la vio nacer no es el mismo que el del lector contemporáneo, hecho que puede determinar que éste no entienda o malinterprete algunos pasajes e incluso el significado del conjunto de la obra. Además, se podría agregar que, al igual que en la conversación el silencio comunica, en la lectura, la ausencia de lo esperado, como mínimo, desconcierta.

Leer tiene que ver, pues, con determinadas expectativas y éstas están determinadas culturalmente; es decir, las expectativas son nuestras expectativas. Pero si profundizáramos un poco más, podríamos matizar diciendo que es posible también que dichas expectativas propias de la cultura a la que pertenecemos estén determinadas por la edad, género, condición social o experiencias vitales del lector, con lo cual la lectura deviene un proceso más complicado, si cabe. Además, leer implica anticipar contenidos, situaciones, respuestas, etc., lo cual lleva, a su vez, a aceptar-confirmar o descartar. Es decir, leer es también un complejo proceso de negociación del significado. Y, además, en literatura nada es inocente, sino que todo obedece a la intención explícita del autor, que oculta o muestra, que comparte o esconde, según su voluntad, por lo que, para entender el mensaje, el lector ha de compartir las coordenadas culturales en las que la obra está inmersa.

\section{THE High Window}

Para ejemplificar lo anteriormente dicho sobre el complejo fenómeno de la lectura, hemos escogido la novela de Raymond Chandler titulada The High Window [1943]. La llamada 'novela policíaca' suele tener detractores, que desprecian este género por considerarlo de escaso valor literario, y defensores, que optan por no generalizar y ponen como ejemplo el buen hacer de Raymond Chandler o P. D. James. En nuestra novela leemos:

It was a cosy sort of office, not too large. There was a built-in upholstered corner seat by the French window and a man in a white dinner-jacket was standing with his back to the room, looking out. He had grey hair. There was a large black and 
chromium safe, some filing-cases, a large globe in a stand, a small built-in bar, and the usual broad heavy executive desk with the usual high-backed padded leather chair behind it.

I looked at the ornaments on the desk. Everything standard and all copper. A copper lamp, a pen set and a pencil tray, a glass and copper ashtray with a copper elephant on the rim, a copper letter opener, a copper thermos bottle on a copper tray, copper corners on the blotter holder. There was a spray of almost coppercoloured sweet peas in a copper vase.

It seemed like a lot of copper.

The man at the window turned around and showed me that he was going on fifty and had soft ash-grey hair and plenty of it, and a heavy handsome face with nothing unusual about it except a short puckered scar in his left cheek that had almost the effect of a deep dimple. I remember the dimple. I would have forgotten the man. I remembered that I had seen him in pictures a long time ago, at least ten years ago. I didn't remember the pictures or what they were about or what he did in them, but I remembered the dark heavy handsome face and the puckered scar. His hair had been dark then.

He walked over to his desk and sat down and picked up his letter opener and poked at the ball of his thumb with the point. He looked at me with no expression and said. 'You're Marlowe?'

I nodded. (Chandler 2011: 143)

El narrador es el detective Philip Marlowe, que ha sido contratado por la señora Elizabeth Wright Murdock para recuperar un valioso doblón que le ha sido robado y para encontrar a su nuera, Linda Murdock, sospechosa del hurto. Sus pesquisas lo llevan al domicilio de Alex Morny, que le confiesa que su mujer se está viendo con un tal Louis Vannier y que lo contrata, a su vez, para que se asegure de que Vannier se aparte de ella. La trama se complica cuando Marlowe encuentra a Linda Murdock cantando en un club nocturno propiedad de Morny.

Analizaremos la escena en la que Marlowe entra en el despacho de Alex Morny, que se inicia con una descripción 'desde afuera' (Pouillon) en la que el narrador ofrece sólo lo que ve: un hombre canoso, de esmoquin, está de espaldas a él frente a una ventana francesa. Hay un escritorio con una silla de cuero de respaldo alto, acolchada, unos archivadores, un mueble bar, una caja fuerte. Esta aparentemente aséptica descripción está 'contaminada' por el adjetivo 'cosy' (acogedora), que contrasta con la formalidad del lugar. Sorprende, además, el efecto de contrapunto que ofrece el término, además de las connotaciones que aporta, ya que es un adjetivo que esperaríamos encontrar en una estancia ocupada por una mujer.

El párrafo siguiente se concentra en la descripción de lo que hay sobre la mesa, que se aborda desde la misma visión 'desde fuera'. Pero en esta ocasión lo que sorprende es la presencia de la aliteración; en concreto, la repetición de la palabra 'cobre'. Así, hay una lámpara de cobre, un cenicero de cobre cuya orilla está decorada con un elefante del mismo 
metal, un abridor de cartas de cobre, un termo de cobre sobre una bandeja de cobre, un porta papel secante con esquinas de cobre y un jarrón de cobre adornado con unos guisantes de cobre. 'Cobre' aparece diez veces sobre un total de sesenta y seis palabras. Pero quizá lo más llamativo sea la segunda oración: Everything standard and copper (Todo estándar y de cobre). La conjunción 'y', que une elementos iguales, enfatiza el valor negativo de 'cobre', que equipara a 'estándar'. Una vez más, se rompe la aparente objetividad del texto, en esta ocasión con la aparición de estos dos elementos: estándar y cobre (término éste que se repite con profusión).

La transición entre el segundo y el tercer párrafo se lleva a cabo mediante un comentario propio de otro tipo de narrador, ya que se ofrece una valoración de lo descrito: It seemed like a lot of copper (Parecía como demasiado cobre).

El párrafo cuatro se centra en la descripción del señor Morny, quien se gira hacia la puerta al oír entrar al visitante. Una vez más, el narrador comunica sólo lo que ve: un hombre de casi cincuenta años, con abundante pelo canoso y una cara atractiva, salvo por una cicatriz en la mejilla izquierda. Sin embargo, esta nueva descripción aparentemente aséptica se ve matizada por ciertos elementos que aportan significado. El pelo canoso es 'suave'; la cara atractiva, 'mofletuda'; la cicatriz parece un hoyuelo. Dos adjetivos y un sustantivo hacen un juicio de valor sobre el personaje. El lector no puede no pensar en las manos regordetas y blandas de Karenin, en Guerra y paz, de Tolstoi, o en la tez tan blanca y las manos cubiertas de anillos de Frederick Fairlie o los pasos sigilosos y leves del conde Fosco, en La mujer de blanco, de Wilkie Collins. Hay a continuación una brevísima transición hacia la segunda parte de la descripción, a partir de una visión 'por detrás', caracterizada por un narrador-personaje que admite recordar el hoyuelo en la cara de Morny, a quien ha visto en el cine. Sin embargo, confiesa que de eso harán unos diez más, más o menos, y que no recuerda el nombre de las películas; sólo su cara morena y mofletuda, la cicatriz y el pelo por aquel entonces oscuro de Morny. Las palabras 'neutras' del narrador comunican algo más que información. Al admitir que recuerda haber visto sus películas hace mucho tiempo (a long time ago), al menos hace diez años (at least ten years ago), que recuerda su cara mofletuda, que lo habría olvidado a no ser por su hoyuelo, está diciendo que Morny era un actor de secunda fila, lo mismo que las películas en las que había trabajado.

En el siguiente párrafo, el narrador vuelve a la visión 'por afuera' para, al igual que una cámara de cine, describir cómo el personaje se acerca al escritorio, coge el abrecartas, lo mira con una mirada inexpresiva y le pregunta si es Marlowe. Nuevamente, la aparente distancia del narrador con respecto a lo que describe se ve contaminada por la mención a la 
cara inexpresiva de su interlocutor e incluso por la pregunta retórica, cuya respuesta conoce de antemano. No le va a la zaga el aludido, que asiente con un movimiento de cabeza ( $I$ nodded).

En el capítulo dedicado a las comunidades lingüísticas presente en su ya clásica obra Language, Bloomfield (1933) menciona una valiosa distinción entre las diferencias de habla locales, es decir, geográficas, y las no locales, de índole social. El estudio de las primeras es el objeto de la dialectología, una de cuyas metas es la identificación y demarcación de isoglosas. Años más tarde Labov (1972) estudió las variaciones intradialectales, es decir, las distintas pronunciaciones de un hablante o de varios en la misma área, dependiendo de su edad, género, nivel social, etc. Según este autor, la evolución de una lengua no puede entenderse sin tener en cuenta la vida social de la comunidad que la habla. Preocupado por cómo abordar el estudio de esas variaciones inherentes a la propia lengua, así como los factores sociales que influían en la misma y en los grupos sociales responsables de la introducción y propagación de los cambios lingüísticos, creó el concepto de 'variable sociolingüística'. La variable sociolingüística es a la pronunciación que constituye la 'norma' lo que el alófono al fonema. Por ejemplo, podemos pronunciar la palabra 'hasta' aspirando la /s/ o pronunciándola. Una u otra pronunciación pueden tener que ver con determinantes geográficos o sociales, pero en ambos casos el interlocutor entiende que se trata de la misma preposición. Así pues, tanto Bloomfield como Labov hablan de una diferencia social en la pronunciación. De hecho, en sociolingüística las categorías de 'variante lingüística' y 'variante sociolingüística' se suelen usar indistintamente para explicar las diferencias en pronunciación. Extrapolando, se podría utilizar el concepto de variante sociolingüística en contextos más amplios que la palabra. Hemos visto cómo Morny saluda al recién llegado con una pregunta retórica: You Marlowe?. Es factible que haya estado esperando su visita, o que le hayan informado de su presencia en la sala. De todos modos, correspondería que lo hubiera recibido con un: 'Entre, por favor, señor Marlowe', 'Encantando de conocerlo, señor Marlowe' o simplemente: 'Adelante'. Pero, en vez de emitir la pregunta completa (Are you Marlowe? [¿Es usted Marlowe?] o, más coloquialmente, You are Marlowe? [¿Usted es Marlowe?]), utiliza una reducción: «You Marlowe?», que aporta información sobre este personaje. Tanto Morny como Marlowe se apartan de la interlocución esperada -y del código social esperado-; el primero, mediante una pregunta retórica y coloquial y el segundo, con un mero movimiento de cabeza. 
Con respecto a Marlowe, el lector de este género literario no puede evitar pensar cómo habrían respondido otros detectives. Por ejemplo, es impensable que Adam Dalgliesh, Comandante de la policía metropolitana londinense, criado en una rectoría en Norfolk y educado en colegios privados y en Oxford y autor de varios libros de poesía, hubiera respondido con un gesto. Lo mismo se podría pensar de Nero Wolfe, un sibarita que se pasa el día leyendo literatura, cuidando de sus orquídeas o degustando los platos que le prepara su chef privado; el mismo que hace esperar a los invitados en una sala antes de informar a Wolfe de su llegada. Para este lector, Marlowe se parece más a Sam Spade, el célebre detective ideado por Dashiell Hammett.

Es posible que el lector también traiga consigo al abordar este texto el recuerdo de otros libros protagonizados por Marlowe o películas como 'El largo adiós' y, cómo no, 'El sueño eterno', en los que Marlowe encarna la figura del anti-héroe, un personaje solitario, soltero y sin familia -al menos, que se sepa-, de mediana edad, bebedor de whisky, pero con unas sólidas convicciones morales a pesar de que su trabajo lo lleva a veces a bordear la ilegalidad. Con todo, a Marlowe también le gusta la poesía -de ahí la conexión con Dalglieshy su apellido coincide con el del dramaturgo inglés homónimo. Tanto Morny como Marlowe se alejan de la conducta esperada y con ello comunican más que lo que dicen sus palabras, siempre y cuando el lector comparta las coordenadas socio-culturales pertinentes.

Si en lingüística -y en tantos otros campos- un fenómeno puede definirse no por lo que es sino por lo que no es, veamos cómo está caracterizado el personaje de Marlowe. Se podría decir que hay un esquema general, que podríamos denominar 'detective de ficción', que contiene información arquetípica sobre dicho personaje. ¿Qué se nos dice de Marlowe? Sabemos que fuma cigarrillos o en pipa (Chandler 2011: 25), que bebe whisky de la marca Four Roses o ginger ale con hielo (Chandler 2011: 77), que tiene una Colt 38, que lleva gafas de sol y que tiene una oficina que parece un cuadro de Hopper: funcional, casi vacía, a excepción de los elementos imprescindibles, sin concesión alguna a la frivolidad. Leemos:

I had an office in the Cahuenga Building, sixth floor, two small rooms at the back. One I left open for a patient client to sit in, if I had a patient client. There was a buzzer on the door which I could switch on and off from my private thinking parlour.

[The reception room] was empty of everything, but the smell of dust. I threw up another window, unlocked the communicating door and went into the room beyond. Three hard chairs and a swivel-chair, a flat desk with a glass top, five green filing-cases, three of them full of nothing, a calendar and a framed licence bond on the wall, a phone, a washbowl in a stained wood cupboard, a hat-rack, 
a carpet that was just something on the floor, and two open windows with net curtains that puckered in and out like the lips of a toothless old man sleeping. The same stuff I had had last year, and the year before that. Not beautiful, not gay, but better than a tent on the beach (Chandler 2011: 23).

Llama la atención la descripción del lugar como un espacio vacío, a excepción del olor a polvo ([The reception room] was empty of everything, but the smell of dust), que puede remitir a su despreocupación por las tareas domésticas, ya que ni siquiera tiene una asistenta, o a su maltrecha economía. Tal vez esta segunda opción sea la adecuada, pues leíamos arriba que en su oficina había una sala de espera, donde esperaba el cliente, de haberlo.

Leemos también que juega al ajedrez, afición solitaria que lo ayuda a concentrarse en sus casos:

The chessmen, red and white bone, were lined up ready to go and had that sharp, competent and complicated look they always have at the beginning of a game. It was ten o'clock in the evening. I was at home at the apartment. I had a pipe in my mouth, a drink at my elbow and nothing on my mind except two murders and the mystery of how Mrs. Elizabeth Bright Murdock had got her Brasher Doublon back while I still had it in my pocket.

I opened a little paper-bound book of tournament games published in Leipzig, picked out a dashing-looking Queen's Gambit, moved the white pawn to Queen's four, and the bell rang at the door (Chandler 2011: 112).

De repente suena el timbre; es el detective Breeze, a quien le confiesa minutos más tarde que de tanto en tanto una partida de ajedrez le permite pensar con claridad acerca de un caso: «Once in a while I fool around with a game here, working things out.» Y agrega que juega viejas partidas que han sido publicadas: «I play over toumament games that have beem recorded and published. There's a whole literature about chess. Once in a while I work out problems» (Chandler 2011: 115).

Por último, se entiende que la visión arquetípica del detective se refiere a una persona preocupada por la justicia. También aquí Marlowe cumple con este requisito. En una conversación que mantiene con el detective Breeze, Marlowe menciona el caso del multimillonario Cassidy, en cuya casa su hijo había sido asesinado de un tiro. Según la prensa y una investigación policial deficiente, el asesino había sido su secretario, pero Marlowe no lo tenía claro. Después de preguntarle qué importa quién le haya disparado, Breeze agrega que el caso está resuelto, a lo que Marlowe le responde preguntándole, a su vez, si en alguna ocasión se había parado a considerar que quizá el secretario tenía madre o hermana o novia y que quizá a ellas sí les gustaría saber la verdad y limpiar su nombre («Did you ever think that 
Cassidy's secretary might have had a mother or a sister or a sweetheart -or all three?») (Chandler 2011: 122). Marlowe le dice que de la misma manera en que él y su ayudante son dueños de sus propias almas, no lo son de la suya («Untilyou guys own your own souls you don’t own mine» Chandler 2011: 122-123). Y remata con el alegato de que él tiene derecho a oír a su conciencia («I have a right to listen to my conscience» Chandler 2011: 123). Con esta actitud, Marlowe ratifica lo que el comisario ha oído acerca de él: que prepara buen café, que se levanta tarde y que su opinión es fiable. Pero matiza que también se dice de él que esto es así siempre y cuando haya cinco testigos independientes que lo atestigüen ([Randal] «says you make good coffee and get up kind of late in the mornings [...] and that we should believe anything you say, provided we can check it by five independent witnesses» Chandler 2011: 115).

Este marco general, que se podría calificar de arquetípico, se halla matizado por información que choca con la idea preconcebida que dicho marco general brinda. Y el lector ha de actualizar, como se dice en informática, subcategorías que lo acercan a otros detectives de ficción. Como hemos dicho, el nombre de nuestro detective tiene connotaciones literarias. Lejos de ser un hecho aislado, éstas están presentes en otras ocasiones y matizan al personaje, creando una subcategoría, un sub-esquema dentro del esquema general al que hemos aludido, que acerca al personaje a detectives como Nero Wolf o el comandante Dalgliesh.

En un diálogo a propósito de la caligrafía de un tal Phillips contenida en su diario, Breeze le comenta que quizá ésta era un pequeño juego, a lo que Marlowe le responde: «Like Pepsy's shorthand» (Como la taquigrafía de Pepys (Chandler 2011: 130). Intrigado, Breeze le pregunta a qué se refiere y nuestro detective le aclara que se trata de un diario que alguien había escrito hacía mucho tiempo usando una taquigrafía propia ( $A$ A diary a man wrote in private shorthand a long time ago» (Chandler 2011: 130), en clara referencia a Samuel Pepys y su famoso diario. En otra ocasión, cuando había ido a visitar a la señora Morny, su perro, un cocker spaniel de nombre Heathcliff se le abalanza y el chófer de la señora Morny exclama: «Here Heathcliff, here Heathcliffs (Aquí Heathcliff; aquí, Heathcliff (Chandler 2011: 41). Sorprendido, Marlowe repite el nombre (Heathcliff?) y el chófer le aclara que así es como se llama el perro. Entonces Marlowe pregunta retóricamente -no sabemos si como broma o con sorna: Whuthering Heights? (¿Cumbres Borrascosas? Chandler 2011: 41), en alusión a la novela homónima, a lo que el chófer contesta que ha vuelto a hablar con segundas, es decir, que no entiende de qué le está hablando. 


\section{LA LECTURA COMO ANTICIPACIÓN}

Una de las características de la lectura es su capacidad para anticipar. Esto no se da en el vacío, sino que, por el contrario, presupone lo que hoy llamaríamos una base de datos que permite no sólo 'encajar' en ella la información, sino también actualizarla y redirigirla hacia otra(s) base(s) de datos. Por otra parte, habría que añadir que dicha sofisticada base de datos es tanto individual como colectiva, es decir, cultural.

Este proceso anticipatorio se da a niveles distintos y todos confluyen en el resultado final; es más, se complementan. Por ejemplo, ante un grupo consonántico /pl/, el lector castellano hablante prevé una serie de posibilidades, como, por ejemplo: plaza, playa, plano, pleno, plica, plausible, plúmbeo. Pero si el contexto aporta datos como la mención de sombrilla, mar, etc., el lector desechará todas salvo una. Es decir, que en este caso sólo se actualizará 'playa'. Lo mismo ocurre a nivel morfológico, sintáctico, etc. Veamos qué ocurre a nivel discursivo, entendiéndose por tal aquel que, a partir de las claves para desambiguar un texto, permite la negociación del significado.

El detective Marlowe va a visitar a Elisha Morningstar, marchante de monedas antiguas. Mediante un procedimiento que en cine se llama 'travelling avanti', con el cual la cámara se acerca al objetivo, el narrador describe el edificio en el que está situada su oficina:

The Belfont Building was eight stories of nothing in particular that had got itself pinched off between a large green and chromium cut-rate suit emporium and a three-storey and basement garage that made a noise like lion cages at feeding time. The small dark narrow lobby was as dirty as a chicken yard. The building directory had a lot of vacant space on it. [ ... ] Opposite the directory a large sign titled against the fake marble wall said $[\ldots]$. There were two open-grille elevators but only one seemed to be running and that not busy. An old man sat inside it slack-jawed and watery-eyed on a piece of folded burlap on top of a wooden stool. He looked as if he had been sitting there since the Civil War and had come out of that badly (Chandler 2011: 58).

Trece líneas plagadas de elementos negativos: «eight stories of nothing in particular» (siete pisos de nada en particular), «the small dark narrow lobby as dirty as a chicken yard» (la pequeña, oscura y estrecha entrada tan sucia como un gallinero), «the building directory had a lot of vacant space on it» (el directorio de empresas tenía un montón de espacios libres), «a fake marble wall» (una pared que imitaba mármol), "only one [open-grille elevador] seemed to be running and that not busy”» (sólo un ascensor parecía estar en funcionamiento, y no se usaba demasiado), «an old 
man $[\ldots]$ with watery eyes $[\ldots]$ looked as if he had been sitting there since the Civil War» (un hombre de edad $[\ldots]$ de ojos acuosos [ ... ] parecía haber estado sentado allí desde la Guerra Civil). Esta primera descripción no anticipa nada bueno del tal señor Morningstar; es más, prepara al lector para la descripción de su propia oficina. Sigue su curso la cámara, que no es sino el narrador que ve a través de los ojos de nuestro detective, el cual describe en primera persona los dos ambientes de que consta la oficina de Morningstar. Veamos el primero:

I turned the knob and went into a small narrow room with two windows, a shabby little typewriter desk, closed, a number of wall cases of tarnished coins in titled slots with yellowed typewritten labels under them, two brown filing-cases at the back against the wall, no curtains at the windows, and a dust-grey floor carpet so threadbare that you wouldn't notice the rips in it unless you tripped over one. [...] Through the door came the small sounds a man makes when he isn't doing anything at all (Chandler 2011: 59).

El lector se encuentra con una pequeña habitación estrecha («a small narrow room»), un pequeño y sucio escritorio, cerrado, sobre el que está situada una desvencijada máquina de escribir («a shabby little typewriter desk, closed»), unas monedas expuestas en la pared, con sendas etiquetas amarillentas («yellowed typewritten labels under [tarnished coins]), dos ventanas sin cortinas («no curtains at the windows») y una raída alfombra gris-polvo («a dusty-grey floor carpet so threadbare»").

Si la descripción negativa del edificio anticipa la descripción también negativa de lo que el lector espera encontrar en la antesala de la oficina, ésta anticipa, a su vez, las características no sólo de la misma, sino de su ocupante:

The inner office was just as small but had a lot of stuff in it. A green safe almost blocked off the front half. Beyond this a heavy old mahogany table against the entrance door held some dark books, some flabby old magazines, and a lot of dust. In the back wall a window was open a few inches, without effect on the musty smell. There was a hat-rack with a greasy black felt hat on it. [On a table there was] a cracked yellow silk handkerchief spotted with ink (Chandler 2011: 59).

La lectura de este párrafo confirma lo esperado, ya que se trata de una habitación pequeña aunque llena de cosas ("the inner office was just as small but had a lot of stuff in it»), entre ellas, una vieja y pesada mesa de caoba («a heavy old mahogany table», unos libros oscuros («some dark bookss》) y unas revistas viejas («some flabby old magarines») y un montón de polvo («a lot of dustı). La ventana situada en la pared posterior está apenas entreabierta, lo que no impide 
que la estancia huela a humedad («a window was open a few inches, without effect on the musty smelli), es decir, a suciedad y abandono. El sombrero que cuelga del perchero está sucio; de hecho, está grasiento («a greasy black. felt hat»). Y sobre la mesa hay un ajado pañuelo de seda amarillo con manchas de tinta («a cracked yellow silk handkerchief spotted with ink»). Ningún elemento positivo, como se esperaba. De todos modos, este párrafo, que describe la oficina del señor Morningstar, es interesante porque constituye la transición desde la descripción física de un lugar a la descripción física de su ocupante. Y todo está impregnado de negatividad. Es más, le lector comprende que es posible que el tal señor Morningstar haya tenido mejores días desde el punto de vista laboral, más éxito en su profesión (la mesa de caoba), que puede que sea una persona instruida (libros oscuros y quizá revistas especializadas) y que su actividad laboral haya ido en declive, al igual que su aspecto personal, como constatará unas líneas más adelante. Con el mencionado 'travelling', el narrador prepara al lector para la caracterización del personaje y el lector puede descodificar la información que se le brinda y anticipar contenido precisamente porque activa los esquemas mentales que le permiten procesar la información.

La cámara se detiene, por último, en Morningstar, a quien se describe de forma aparentemente aséptica como un hombre de edad, que lleva un traje posiblemente pasado de moda (con grandes solapas y demasiados botones) y de aspecto desaseado, como prueba el sucio cuello 'hoover' con el que remata la camisa. Utilizamos la expresión 'aparentemente aséptica' porque en literatura nada es aséptico ni ocurre porque sí, sino que todo obedece a la intención del autor.

In the swivel-chair at the desk sat an elderly party in a dark grey suit with high lapels and too many buttons down the front. [ ... A Hoover collar which no decent laundry would have allowed on the premises nudged his Adam's apple (Chandler 2011: 60).

Además de la capacidad para preveer/anticipar información, como hemos visto, el lector puede valerse de información para 'cerrar' el esquema que ha abierto para descodificar la información. Se trata de elementos anafóricos, de alto valor cohesionador del relato. Unas páginas más adelante, Marlowe le espeta lo siguiente al ocupante de la infame oficina:

[The people who wanted to sell the coin] knew that the building where you had your office was a shabby dump where anything could happen (Chandler 2011: 64). 
El valor sintetizador y anafórico de este texto, que describe el inmueble con la frase 'desgastado vertedero donde todo puede ocurrir' («a shabby dump where anything could happen») refuerza la cohesión del relato.

\section{LA DECODIFICACIÓN DEL HUMOR Y LA IRONÍA}

Hemos dejado para el final la referencia al humor ácido de Marlowe. El humor es un recurso complejo, que trasciende el valor denotativo de las locuciones. De hecho, su comprensión deriva del valor connotativo de las mismas. Hemos hablado arriba de los 'esquemas de contenido' de Carrell y hemos dicho que en muchas ocasiones dichos esquemas son estructuras mentales determinadas culturalmente. Veamos algunos ejemplos presentes en la novela.

Al comienzo de la trama, Philip Marlowe acude al domicilio de la señora Elizabeth Murdock, que ha contratado sus servicios como detective. Hay en la puerta un llamador de metal a los pies de la figura de un jinete negro en pantalones de montar blancos, chaqueta verde, gorra roja y fusta, con cara triste y expresión de haber estado esperando allí durante mucho tiempo -como tendrá que hacer nuestro detective. Llama y al cabo de unos minutos aparece una doncella, quien con la puerta apenas entreabierta y sin mediar palabra le lanza una mirada poco amistosa con sus ojos pequeños y brillantes. Marlowe le dice cuál es el propósito de su visita y la doncella le cierra la puerta en las narices. Marlowe se limita a pensar que quizá habría sido una mejor idea haber llamado a la puerta trasera y, después de darle al jinete unas palmaditas en la cabeza, le dice en voz alta: «Brother [ ... ] you and me both» (Chandler 2011: 3) (Hermano, [ ... ] tanto tú como yo). Es decir, las personas de color y los criados o repartidores no llaman a la puerta principal, sino a la de servicio.

$\mathrm{Al}$ cabo de un buen rato esperando fuera, la misma criada lo hace pasar. Después de describir el amplio recibidor, Marlowe sintetiza lo que ve con un: «mucho dinero y gastado en balde» («A lot of money and all wasted» (Chandler 2011: 3). Una vez más, es la información que trae consigo el lector la que le permite entender la falta de modales de la criada -y, seguramente, de la propietaria-, pues lo correcto habría sido hacerlo pasar de inmediato y decirle que esperara en el recibidor. La ironía se deriva en esta ocasión del mensaje subliminal que achaca el mal gusto de la dueña de casa al rápido ascenso social de los Murdock, una familia de nuevos ricos. 
En otra ocasión en el curso de sus pesquisas, Marlowe acude a la oficina de un tal señor Anson, situada en Bunker Hill, una zona venida a menos y depauperada, de rentas bajas. A la entrada está el encargado, quien le dice que ese es un lugar respetable. Con sorna, Marlowe le responde que también es silencioso y agrega que arriba casi se puede oír el chillido de un águila. Su interlocutor se sonríe y contesta que no es fácil que se divierta. Marlowe entonces le dice: «Just like Queen Victoria» (Como la reina Victoria) y el portero contesta «I don't get it» (No lo cojo). Es obvio, pues, que desconoce la solemnidad de la casa real británica de la época victoriana e ignora quién era la reina Victoria de Inglaterra.

En la segunda visita al domicilio de Marlowe, el ayudante de Breeze, Sprangler, coge el arma que está sobre la mesa y le dice que hay un poco de polvo en el cierre del cañón, lo que indica que el arma no ha sido disparada recientemente, a lo que nuestro detective contesta: «What did you expect? Rubies?» (Chandler 2011: 113) (¿Qué esperaba? ¿Rubíes?). En este ejemplo, la ironía proviene de la respuesta no esperada, que habría sido desde un 'sí' hasta 'es verdad' o 'ahí tiene la prueba'.

En el curso de su investigación, Marlowe informa a la patrulla de vigilancia situada a la entrada de Idle Valley que se dirige al Idle Valley Club. Se le dan instrucciones para llegar al sitio y se le comunica que tiene que informar de su llegada al guardia de seguridad, ya que en caso contrario, el cuerpo de seguridad iniciaría su búsqueda por el recinto. A lo que Marlowe contesta: «And they call this a democracy?» (Chandler 2011: 136) (¿Y a esto llaman democracia?). A continuación, el guardia le explica otro de los motivos por los que hay tanta seguridad. Además de ser una zona residencial de gente acomodada, hay otros 'inquilinos': «I knew a fellow belonged to the John Reed Club». (Chandler 2011: 136) (Sabía que un tipo pertenecía al club John Reed), a lo que Marlowe contesta con un simple: «Tovarich» (Camarada). El humor ácido de Marlowe proviene, por una parte, del contraste entre la fuerte seguridad privada de un recinto en un país que hace gala de su democracia y, por otra, de la relación entre John Reed-tovarich, para cuya comprensión hay que compartir las coordenadas histórico-culturales pertinentes. Sólo quien sepa que John Reed fue un periodista norteamericano que siguió in situ el devenir de los primeros días de la revolución de Octubre, que escribió un libro titulado Los cien días que conmocionaron al mundo, que fue miembro de la Tercera internacional y que está enterrado en la Plaza Roja de Moscú es capaz de sonreír ante la respuesta de Marlowe.

Hemos analizado brevemente unos ejemplos de humor e ironía en los que la clave para su decodificación deriva de elementos históricos (John Reed, la reina Victoria), del saber 
estar (la espera a la puerta), de la respuesta socialmente esperada (la contestación a propósito de los vestigios de pólvora) y del racismo/clasismo (la figura pintada en la puerta de la mansión Murdock), siempre y cuando, insistimos, el lector posea el esquema mental previo que le permite entender el alto valor connotativo de los comentarios y acceder al ácido sentido del humor de Marlowe.

\section{CONCLUSIÓN}

Al leer se abren, pues, una serie de esquemas presentes en la mente del lector. Este proceso se da desde los niveles más ‘simples’ hasta los más sofisticados. Por ejemplo, en la casa de empeños Marlowe dice lo siguiente: «I gave my true name and address» (Chandler 2011: 107). De forma muy rápida, el lector es capaz de anticipar lo que espera encontrar después de la referencia al nombre, desde «age» (edad) a «address» (dirección) y es el contexto lo que le permite actualizar sólo la segunda opción, hecho que confirma al leer a continuación que vive en el 1624 de la Avenida North Bristol, Holywood. Al ver la dirección, el dueño le contesta: «You are living in that district and you are borrowing fifteen dollan» (Chandler 2101: 107). El lector puede entender que el barrio en cuestión es una zona donde viven personas de alta posición social, pero sólo aquel que conozca el barrio o que haya estado allí sabrá que se trata de una zona residencial de Hollywood muy exclusiva donde se hallan mansiones como la habitada en su día por la actriz Joan Crawford.

En otra ocasión, Marlowe vuelve al edificio Belfont y comenta: «The same watery-eyed relic sat motionless (Chandler 2011: 194). El término 'relic' tiene varias acepciones y significa 'objeto, costumbre o tradición que ha sobrevivido desde el pasado y que aún sigue en vigor', 'objeto que se conserva porque pertenece a una persona famosa o a un acontecimiento importante del pasado', 'el vestigio de algo hecho o construido en el pasado', y 'el cuerpo de un santo o algo íntimamente ligado a un santo y que se cree que es sagrado'. Dicho en términos lógicos, el lector, ignorando la diferencia específica, se apoya en el género próximo de las acepciones, es decir, en la idea de 'antiguo' para comunicar su apreciación acerca de la avanzada edad del ascensorista. En una de sus visitas a la señora Murdock, Marlowe ve en la mesita una botella de oporto y comenta: «She was pouring herself a slug from what looked like the same port bottle but was more probably a grandchild» (Chandler 2011: 159). Aparentemente la presencia del término «nieta» en este contexto está fuera de lugar. Sin embargo, el lector ha 
de acudir a una asociación de ideas para llegar a la conclusión de que es posible que se trate de una tercera botella -la primera sería la que estaba bebiendo en la primera cita y a ésta habrían seguido una segunda -la hija- y una tercera -la nieta, en alusión a la afición a la bebida de la señora Murdoch.

Según hemos dicho, los esquemas emergentes en la mente del lector también están condicionados por factores como la edad, el género, la ideología, etc. Sobre la mesa del señor Morny se halla un porta papel secante con esquinas de cobre (copper corners on the blotter holder). Es posible que el lector de una cierta edad active el esquema correspondiente, en el que es lógico presumir la existencia de este tipo de papel sobre la mesa de un escritorio; quizá lo haya usado de niño para secar la tinta al hacer unos deberes escolares. Pero es posible que el lector joven no encuentre en su base de datos información alguna sobre este tipo de papel. Con todo, el no entender este detalle no le impide seguir leyendo y comprender el contexto más amplio, pero sí lo priva de información.

Agotado al cabo de un día complicado, Marlowe está exhausto y el narrador comenta: «But even the Pinkertons have to sleep, and Marlowe needed far, far more sleep than the Pinkertons» (Chandler 2011: 158). Si el lector carece de esta información en su base de datos, es posible que no entienda la referencia a estos Pinkerton. Es posible, asimismo, que abra y cierre sucesivamente casilleros correspondientes a nombres propios, otros personajes de la novela, etc. y que al no encontrar dónde colocar esta referencia pase por encima de ella al considerarla un detalle prescindible. Sin embargo, es seguro que la mención a los Pinkerton active en el lector de una cierta edad o ideología o en el que conozca algo acerca de la historia contemporánea de los Estados Unidos la información pertinente sobre la agencia de rompehuelgas Pinkerton, de tan funesta memoria en la historia de aquel país. Es más, recordará que su logo era un ojo con el lema 'Never sleep' (Nunca dormimos), que inspiró el mote de 'private eye' con el que más tarde se conocería a todos los detectives privados.

En ocasiones, la comprensión se da por asociación a una imagen, nombre, concepto, situación, artilugio, etc. Una noche Marlowe decide acercarse a la casa de Vannier en Escamillo Drive. Al llegar llama al timbre y éste resuena en lo que parece una casa deshabitada: «I rang the bell, and it rang with that remote sound of a bell ringing at night in an empty house» Después de dar una vuelta alrededor del inmueble, se decide a entrar; enciende una lámpara: «I went over to switch a lamp on [ ... ] The lamp had a big bulb inverted in a porcelain glass bowl. You could get three different intensities of light. I clicked the button switch around until I had all there was» (Chandler 2011: 217). La lectura de este breve texto abre distintas ventanas: la del sonido 
del timbre en una casa vacía, la del modelo de lámpara y, sobre todo, la del tipo de interruptor. Sólo quien conozca este tipo de interruptor comprenderá que Marlowe ha de pulsarlo y luego girarlo hasta obtener la máxima potencia de luz; de desconocerlo, puede no entender cómo funciona la lámpara. Puede tratarse de un ejemplo baladí, pero ilustrativo de las diversas asociaciones que se dan en la lectura y que dependen de información previa.

Casi al final de la novela, Marlowe llama al Dr. Carl Moss porque la secretaria de la señora Murdock, la señorita Merle Davis, ha ido a visitarlo para confesar un supuesto crimen y ha sufrido una crisis nerviosa. Marlowe le pide que contrate a una enfermera para que cuide de ella; él buscará un sitio para pasar la noche. El doctor le contesta: «Phil Marlowe [ ... ] The shop-soiled Galahad». En este ejemplo, la asociación se establece con un personaje literario, uno de los caballeros del rey Arturo: Sir Galahad, conocido por su gallardía. Al igual que el héroe artúrico, Marlowe socorre a una dama en apuros y defiende su honor. Será un Galahad un poco venido a menos, pero igual de caballeroso, en opinión del Dr. Moss. Como se ha visto, los nombres propios juegan un papel interesante en la comprensión por asociación, tanto si se trata de asociaciones de tipo literario como si no, como se verá a continuación. Marlowe visita al señor Morny en su club nocturno: Idle Valley Club (Club del Valle indolente). Si la traducción al castellano de The High Window no traduce el nombre del establecimiento y mantiene el nombre original, sólo el lector que sepa inglés recibirá la misma información que el lector de habla inglesa, es decir, ambos entenderán que se trata de un nombre que define a la perfección al club de Morny, un local donde se juega, se bebe y se pierde el tiempo. En otras ocasiones, la comprensión deriva de la acumulación de información, proceso en el cual la asociación y la catáfora juegan un papel muy interesante. En una de sus visitas a la mansión Murdock, Merle Davis le confiesa a Marlowe que ella había sido la secretaria del primer esposo de la señora Murdock, muerto en trágicas circunstancias; de hecho, Horace Bright se había caído por una ventana y Merle había presenciado aparentemente el hecho. Agrega que la viuda se había portado muy bien con ella desde entonces, la había contratado como su secretaria personal y la protegía. Los hechos apuntan a la posible implicación de Merle en dicha muerte, cuyas circunstancias se desconocen. Al poco tiempo se produce la visita de Merle a Marlowe de la que hemos hablado arriba, en la que una Merle presa del pánico le confiesa que ha matado a un tal Vannier, cosa que nuestro detective se resiste a creer. Marlowe se dirige a la casa de éste y al llegar se encuentra con el cuerpo sin vida de Vannier. Aunque todo apunta a un suicidio, Marlowe no cree que la víctima haya acabado con su vida; sospecha, más bien, que se trata de una puesta en escena. Inspecciona la habitación y repara 
en los cuadros que hay en la pared: unas acuarelas y unos grabados antiguos. En uno de los cuadros se ve a un hombre asomado a una ventana alta. Leemos:

The touch of the idea at first was so light that I almost missed it and passed on. A touch of a feather, hardly that. The touch of a snowflake. A high window, a man leaning out - a long time ago. $[\ldots]$. Out of a high window a long time ago - eight years ago - a man leaning - too far - a man falling - to his death. A man named Horace Bright( Chandler 2011: 221).

Tanto Marlowe como el lector establecen sendas asociaciones con hechos precedentes: la confesión de Merle de haber presenciado al muerte del señor Bright y la confesión de haberle llevado dinero a Vannier la noche en la que se lo encontró muerto en su casa de Escamillo Drive. La reacción inmediata del lector -y cómo no, de Marlowe- es preguntarse quién está comprando el silencio de Vannier y por qué. A continuación, Marlowe retira una acuarela de la pared y le da la vuelta: en el reverso están escritas unas fechas y unas cantidades recibidas a lo largo de ocho años; la mayoría son pagos por valor de quinientos dólares, aunque también hay alguno de setecientos cincuenta y dos de mil. Llama la atención de nuestro detective el hecho de que el cartón en el reverso del cuadro no está del todo sujeto al marco y cuando lo retira encuentra un sobre blanco. Cuál no será su sorpresa al hallar dos copias de una misma foto y un negativo, que muestran a un hombre asomándose a una ventana, gritando, y una mujer detrás. Pero el hombre no estaba apoyado en la ventana; de hecho, estaba cayendo por la ventana.

Tenemos, pues, un esquema elemental en el que una persona ha resultado muerta al caer por una ventana alta. Tenemos, asimismo, a un testigo y quizá autor del crimen: Merle. Tenemos también un posible móvil: recodemos que Vannier había chantajeado a la señora Murdock a raíz de unas fotos comprometedoras en relación con la muerte de su marido. Este esquema se ha ido gestando por acumulación de información, a partir de un primer esquema que podríamos denominar 'arquetípico'. Pero la labor de Marlowe nos dará nuevas pistas que harán que tengamos que readaptarlo, renegociarlo. Porque la lectura es negociación del significado. Es decir, que los esquemas no siempre son compartimentos estancos, sino que, por el contrario, se (re)construyen a medida que leemos. Unas páginas más adelante, nuestro detective recompone el esquema inicial al resolver el caso: la figura femenina detrás de Bright no era Merle, sino su propia esposa, que había cometido el asesinato para quedarse con los cincuenta mil dólares del seguro de vida de su marido, que hacía poco se había arruinado, y había estado haciéndole creer a la pobre secretaria que la culpable había sido ella. 
Esperamos que los ejemplos analizados sirvan para ilustrar el hecho de que al leer se activan simultáneamente una serie de esquemas o 'pantallas' y éstas se activan/desactivan sucesivamente a medida que el lector acepta o rechaza las opciones que se le presentan. Varios esquemas se mantienen abiertos al mismo tiempo, a la par que otros se desactivan, recomponen, descartan o complementan entre sí. La lectura es un acto de negociación del significado, una actividad bidireccional entre el propio texto y el lector, que trae consigo información previa a la hora de leer. Esta información contenida en esquemas previos es múltiple y el proceso de comprensión, sofisticado. La lectura es una actividad muy compleja dominada por el cerebro humano y de la cual sólo se conocen unos rudimentos. Es posible que la teoría de los esquemas arroje algo de luz a un proceso en el que la vista, la memoria, el reconocimiento, la experiencia, las lecturas previas, etc. tienen un papel primordial. Ya no decimos en nuestros días que las cualidades de lo que vemos entran en el ojo por el aire, pero sí que la lectura está relacionada con la percepción, que constituye un acto voluntario de (re)conocimiento que permite al sujeto trascender lo que ve y descifrarlo, es decir, leer. También sabemos que es el cerebro el encargado de garantizar este proceso; es más, sabemos en qué zonas se alojan la memoria, la atención, el lenguaje, las funciones visoperceptivas, etc. Toda esta información, todas estas funciones alojadas en distintas zonas del cerebro permiten acceder a algo que no ha perdido ese halo mágico como es la lectura y descifrar «aquello que la escritura sugiere mediante indicios y sombras», como decía poéticamente Ibn-al-Haytan (Manguel: 2005: 91). 


\section{BIBLIOGRAFÍA}

Bartlett, Frederick (1932): Remembering, Cambridge, Cambridge University Press.

Bigot, Margot (2010): «Hjelmslev: Una reelaboración del signo», en Apuntes de Lingüistica antropológica (2010): 71-85. En http://rehip.unr.edu.ar (último acceso: 19/6/19).

Bloomfield, Leonard (1973): Language, London, George Allen \& Unwin.

Brown, Gillian, George Yule (1983): Discourse Analysis, Cambridge, Cambridge University Press.

Carrell, Patricia (1983): «Some Issues in Studying the Role of Schemata or Background Knowledge in Second Language Comprehension», en Reading in a Foreign Language, University of Aston, Birmingham, Vol. 1, No 2: 81-92.

Chandler, R.aymond (2011): The High Window, London, Penguin.

Gregory, Michael, Susanne Carroll (1978): Language and Situation: Language Varieties and their Social Contexts, Londres, Henley \& Boston, Routledge \& Kegan Paul.

Grice, Herbert (1975): «Logic and Conversation», en Cole, Peter, Jerry Morgan (eds.) Syntax and Semantics 3: Speech Acts, New York, Academic Press: 41-58.

Halliday, Michael Alexander Kirkwood, Ruqaiya Hasan (1976): Cohesion in English, London, Longman.

Halliday, Michael Alexander Kirkwood, Ruqaiya Hasan (1985): Language, Context and Text: Aspects of Language in a Social Semiotic perspective, Oxford, Oxford University Press.

Labov, William (1972): «Some Principles of Linguistic Methodology», en Language in Society, Vol. 1, Cambridge, Cambridge University Press: 97-120.

Manguel, Alberto (2005): Una bistoria de la lectura, Barcelona, Ramdon House Mondadori.

Minsky, Marvin (1975): «A Framework for Representing Knowledge», en Winston, Patrick (ed.), The Psychology of Computer Vision, New York, McGraw Hill: 211-277.

Moss, Aaron. «¿Por qué rompemos un vaso en una boda judía? », en http://es.chabad.org, (último acceso: 18/6/19).

Pouillon, J. (1970): Tiempo y novela, Buenos Aires, Paidós.

Rumelhart, David (1984): «Understanding Understanding», en Flood, J. (ed.) Understanding Reading Comprehension: Cognition, Language and the Structure of Prose, International Reading Association, USA: 1-20. 


\section{an}

üe Actio nova: REVISTA DE TEORÍA DE LA LITERATURA Y LITERATURA COMPARADA, no 3, pp. 166-193 DOI: https://doi.org/10.15366/actionova2019.3.008

Sacks, Harvey (1984): «Notes on Methodology», en Atkinson, John; John Heritage (eds.) (1983), Structures of Social Action: Studies in Conversation Analysis, Cambridge, Cambridge University Press: 2-27.

Sanford, Antony, Simon Garrod (1982): Understanding Written Discourse: Explorations in Comprehension Beyond the Sentence, Chichester, New York, John Willey \& Sons.

Schank, Roger, Robert Abelson (1977): Scripts, Goals and Understanding, Oxford, England, Lawrence Erlbaum Associates.

Steiner, G. (1998): After Babel: Aspects of Language and Translation, Oxford, Oxford University Press.

Sperber, Dan, Wilson, Deidre (1984) «Pragmatics: An overview», en: Georges, Susan

(ed.) From the Linguistic to the Social Context, Bologna, Cooperativa Libraria Universitaria Editrice. 21-41.

Wikipedia: «Agencia Nacional de Detectives Pinkerton» 
SOBRE LA AUTORA

\section{Beatriz Giudici Fernández}

Licenciada en Filología Hispánica (Literatura) y Filología Inglesa por la Universidad de Oviedo.

Master en Lingüística por la Universidad de Aston - Birmingham (Gran Bretaña).

Doctora en Traducción e Interpretación por la Universidad de León.

Catedrática de Bachillerato (Inglés), actualmente jubilada.

Profesora/tutora en diversos cursos de traducción en EE.UU.

Traductora.

La autora ha publicado diversos artículos sobre Teoría de la Literatura y sobre Traducción y un libro sobre El Maestro y Margarita, de M. A. Bulgakov.

No Orcid 0000-0002-0075-0974

Contact information:

Correo electrónico: giudici@telecable.es 